\title{
Hypoxia-induced microRNA-155 promotes fibrosis in proximal tubule cells
}

\author{
SHENPING XIE, HUAIZHOU CHEN, FEI LI, SHU WANG and JUNJUN GUO
}

\author{
Renal Transplantation and Dialysis Center, 181st Hospital of Chinese People's Liberation Army, \\ Guilin, Guangxi 541002, P.R. China
}

Received March 23, 2014; Accepted September 29, 2014

DOI: $10.3892 / \mathrm{mmr} .2015 .3327$

\begin{abstract}
Hypoxia has been considered to be a significant microenvironmental factor in promoting renal fibrosis, which causes progressive kidney disease and renal allograft failure. Previous studies have demonstrated versatile functions of miR-155 in hypoxia and fibrosis of the lung and liver. However, it is unclear whether miR-155 is able to regulate renal fibrosis and what the detailed mechanisms of this may be. In the current study, we focused on the interaction of miR-155/hypoxia-inducible factor 1 alpha (HIF-1 $\alpha$ ) and the effects of miR-155 on fibrosis in hypoxic HK-2 cells. Analysis of the expression of miR-155 and fibrosis-associated cytokines revealed upregulated miR-155, increased transforming growth factor beta 1 (TGF- $\beta 1$ ) and alpha-smooth muscle actin, and decreased E-cadherin in hypoxic HK-2 cells. Further study demonstrated that miR-155 played a positive role in regulating HIF-1 $\alpha$ and vice versa. Moreover, the data illustrated the synergistic effects of upregulated miR-155 on fibrosis by gain-of-function and loss-of-function methods in hypoxic HK-2 cells. Notably, the results also revealed that miR-155 had the ability to modulate TGF- $\beta 1$ and the process of epithelial-mesenchymal transition (EMT). In conclusion, this study not only demonstrated that hypoxia-induced miR-155 was a pro-fibrotic cytokine which was positively regulated by HIF-1 $\alpha$, but also revealed that miR-155 promoted the fibrosis of proximal tubule cells by regulating both TGF- $\beta 1$ and the process of EMT under hypoxia.
\end{abstract}

\section{Introduction}

Hypoxia is considered to be a significant microenvironmental factor in the promotion of tissue fibrosis, which results in organ dysfunction in diseases including systemic sclerosis,

Correspondence to: Dr Junjun Guo, Renal Transplantation and Dialysis Center, 181st Hospital of Chinese People's Liberation Army, 1 Xinqiaoyuan Road, Xiangshan, Guilin, Guangxi 541002, P.R. China

E-mail: guojj181@163.com

Key words: microRNA, microRNA-155, fibrosis, hypoxia liver cirrhosis, cardiac fibrosis, idiopathic pulmonary fibrosis and progressive kidney disease (1-4). The development of interstitial fibrosis, which is caused by ischemia or hypoxia, is a common complication and a leading cause of graft failure following kidney transplantation $(3,5)$. Therefore, the protection of cells or tissues against fibrosis plays an essential role in the management of diseases, including in patients undergoing kidney transplantation.

Renal fibrosis, particularly tubulointerstitial fibrosis, results in a final outcome of renal failure (6). Renal tubulointerstitial fibrosis undergoes the processes of tubular atrophy, myofibroblast accumulation and extracellular matrix (ECM) deposition, in which fibroblasts are the primary producers of ECM components $(3,7)$. The process by which epithelial cells are converted into myofibroblasts and matrix-producing fibroblasts is known as epithelial-mesenchymal transition (EMT), which plays a key role in the progression of renal interstitial fibrosis (8-10). This involves the replacement of E-cadherin, which is lost gradually, by alpha-smooth muscle actin ( $\alpha$-SMA), which is the main mesenchymal marker in EMT $(11,12)$. With regard to the regulation of EMT, transforming growth factor beta (TGF- $\beta$ ) was characterized as a key mediator (13). TGF- $\beta 1$ not only stimulates the production of collagens, fibronectin and proteoglycans by myofibroblasts, but also triggers its own production $(14,15)$. It was thus speculated that therapies that targeted the TGF- $\beta 1$ pathways may provide effective strategies to slow the progression of fibrosis (3).

MicroRNAs (miRNAs), composed of 22 nucleotides, regulate $\sim 60 \%$ of gene expression by targeting the 3'-untranslated regions (3'-UTRs) to result in negative modulation of relevant mRNA expression (16). miRNAs have a significant effect not only on cellular biology and pathophysiological regulatory pathways, but also on the pathogenesis of fibrosis (17-19). Previous articles have reported that certain miRNAs function as regulatory factors in various fibrotic disorders; for example liver cirrhosis, cardiac fibrosis and idiopathic pulmonary fibrosis (20-22). In renal fibrosis, miR-200a and $\mathrm{miR}-449 \mathrm{a} / \mathrm{b}$ are characterized as anti-fibrotic factors due to their repression of the expression of TGF- $\beta$ and ECM synthesis, respectively (23). Certain miRNAs play pro-fibrotic roles in renal fibrosis, including miR-21 and miR-192 $(24,25)$. Therefore, the properties of miRNAs involved in the regulation of renal fibrosis are unclear. It is documented that miR-155 is abnormally expressed in renal cancer, end-stage 
renal disease and renal transplantation undergoing acute cellular rejection (26-28). Most significantly, a study by Pottier et al reported the effect of miR-155 expression level on lung fibrosis (29). In accordance with these previous studies on the functions of miR-155, interest has been aroused as to whether miR-155 is involved in hypoxia-associated renal fibrosis.

In the current study, proximal tubule cells (HK-2 cells) were exposed to hypoxia. Then, the expression of miR-155 and fibrosis-associated factors was analyzed. Next, we investigated the association of miR-155 and HIF-1 $\alpha$ to reveal the effect of hypoxia on the expression of miR-155. Finally, the study focuses on the mechanisms involved in the correlations between miR-155 and fibrosis in hypoxic tubule cells.

\section{Materials and methods}

Cell culture. Human renal proximal tubule (HK-2) cells were acquired from the American Type Culture Collection (ATCC; Manassas, VA, USA) and cultivated in Dulbecco's modified Eagle's medium supplemented with $10 \%$ fetal bovine serum at $37^{\circ} \mathrm{C}$ in humidified $5 \% \mathrm{CO}_{2}$. The cells were subcultured at $80 \%$ confluence by $0.05 \%$ trypsin with $0.02 \%$ EDTA. After cultivating to $\sim 70 \%$ confluence, cells were treated with hypoxia in Anoxomat chambers (Mart Microbiology, Lichtenvoorde, Netherlands) for physiological hypoxia $\left(5 \% \mathrm{O}_{2}\right)$ or normoxia $\left(21 \% \mathrm{O}_{2}\right)$ at $37^{\circ} \mathrm{C}$ for the indicated times.

Cell treatments. miR-155 mimics (miR-mimics), anti-miR of miR-155 (anti-miR) (GMR-miR ${ }^{\mathrm{TM}}$, Shanghai, China) and miRNA control (control) were purchased from GenePharma (GenePharma, Shanghai, China) (30). HK-2 cells were transfected with miR-mimics $(20 \mathrm{nM})$, miRNA control or anti-miR $(50 \mathrm{nM})$ using Lipofectamine 2000 (Invitrogen, Carlsbad, CA, USA). HIF-1 $\alpha$ siRNA (Santa Cruz Biotechnologies, Santa Cruz, CA, USA) was used to knock down the HIF-1 $\alpha$ genes (HIF-1 $\alpha$-si), and non-specific siRNA (Santa Cruz Biotechnologies) was used as a negative control (si-control). Transfection was conducted using Lipofectamine 2000 according to the manufacturer's instructions. After $16 \mathrm{~h}$ transfection or incubation with the TGF- $\beta$ type I receptor kinase inhibitor LY2157299 (Selleckchem, Houston, TX, USA) at $5 \mu \mathrm{M}$, cells were treated with hypoxia or normoxia for the indicated times.

RNA extraction and reverse transcription-quantitative polymerase chain reaction ( $R T-q P C R)$. Total mRNA was extracted from cells using RNeasy kits (Qiagen, Valencia, CA, USA). RT-qPCR was performed on an ABI 7500 with a SYBR Premix Ex Taq ${ }^{\text {TM }}$ kit (Takara Bio Inc., Shiga, Japan). GAPDH was used as the internal control. For quantitation of miR-155, miRNA extraction was conducted using the mirVana miRNA isolation kit (Ambion, Austin, TX, USA). The mirVana qRT-PCR miRNA detection kit (Ambion) was used to quantify the expression level of miR-155, and U6 small nuclear RNA was used as the internal control. Data were normalized using the $2^{-\Delta \Delta C t}$ method for relative quantification. The used primers were: E-cadherin: F-TTGCAAATTCCTGCCATTC, R-GCTGGCTCAAGTCAAAGTCC; $\alpha$-SMA: F-CTGTTC CAGCCATCCTTCATC, R-GCTGGCTCAAGTCAAAG TCC; TGF- $\beta 1$ : F-TGAACCGGCCTTTCCTGCTTCTCATG,
F-GCGGAAGTCAATGTACAGCTGCCGC; HIF-1 $\alpha$ : F-ATCGCGGGGACCGATT, R-CGACGTTCAGAACTT ATCTTTTTCTT; GAPDH: F-GGAGTCAACGGATTT GGTC, R-GGAATCATTGGAACATGTAAAC.

Western blot analysis. Proteins were collected and subjected to $10 \%$ SDS-PAGE by centrifugation at $12,000 \mathrm{rpm}$ for $30 \mathrm{~min}$ at $4^{\circ} \mathrm{C}$ after HK-2 cells were lysed. Then, proteins were isolated by electrophoresis and transferred onto nitrocellulose membranes using the transfer buffer. The membrane was blocked with $2 \%$ bovine serum albumin to prevent non-specific background binding. Immunoblotting was performed using human monoclonal E-cadherin antibody, $\alpha$-SMA antibody, TGF- $\beta 1$ (Cell Signaling, Inc., Danvers, MA, USA), anti-HIF-1 $\alpha$ (BD Biosciences Inc., Franklin Lakes, NJ, USA) and anti- $\beta$-actin (Sigma, St. Louis, MO, USA). Goat anti-mouse IgG or goat anti-rabbit IgG (Pierce Biotechnology, Inc., Rockford, IL, USA) was utilized to visualize the results, and ECL detection systems (Supersignal West Femto, Pierce) were used for the assay.

Statistical analysis. Independent experiments were performed at least in triplicate. Data were normalized to the mean \pm standard deviation. Comparisons between two groups were made using Student's t-test. ANOVA was utilized to compare differences of multiple samples. SPSS 18.0 (SPSS, Inc., Chicago, IL, USA) was used for general statistical analysis. $\mathrm{P}<0.05$ was considered to indicate a statistically significant difference.

\section{Results}

Hypoxia induces overexpression of miR-155 and promotes fibrosis in proximal tubule cells. To investigate the hypoxic response of proximal tubule cells, HK-2 cells were exposed to hypoxia (5\% oxygen) for $0,24,48$ and $72 \mathrm{~h}$. The relative expression of miR-155 was assessed at the different time points, as shown in Fig. 1A. The data reveal that the expression of miR-155 was significantly stimulated by hypoxia in HK-2 cells after $48 \mathrm{~h}$ exposure, in contrast with normoxia $(\mathrm{P}=0.033$ at $48 \mathrm{~h}$ and $\mathrm{P}=0.012$ at $72 \mathrm{~h}$ ).

Next, to determine the effects of hypoxia on fibrosis in proximal tubule cells, the expression levels of E-cadherin, $\alpha$-SMA and TGF- $\beta 1$, which are well characterized as biomarkers in the process of renal interstitial fibrosis, were examined at the same time in hypoxic HK-2 cells. The expression of E-cadherin mRNA was gradually reduced and reached a significant difference at $48 \mathrm{~h}(\mathrm{P}=0.027)$ and $72 \mathrm{~h}(\mathrm{P}=0.009)$ (Fig. 1B). The expression of $\alpha$-SMA and TGF- $\beta 1$ mRNA was enhanced gradually with increasing time and was significant at $72 \mathrm{~h}$ for hypoxia ( $\mathrm{P}=0.049$ and $\mathrm{P}=0.017$, respectively; Fig. $1 \mathrm{C}$ and D). Similarly, $\alpha$-SMA and TGF- $\beta 1$ protein expression was increased, while the expression of E-cadherin protein was shown to be decreased in HK-2 cells on hypoxic exposure at $72 \mathrm{~h}$ by western blot analysis, as shown in Fig. 1E. All the data reveal that hypoxia induced overexpression of miR-155 and promoted fibrosis in proximal tubule cells.

miR-155 is modulated by HIF-1 $\alpha$ under hypoxia. We analyzed whether the upregulated miR-155 was associated with HIF-1 $\alpha$, which plays a vital role in the cellular response to hypoxia. 

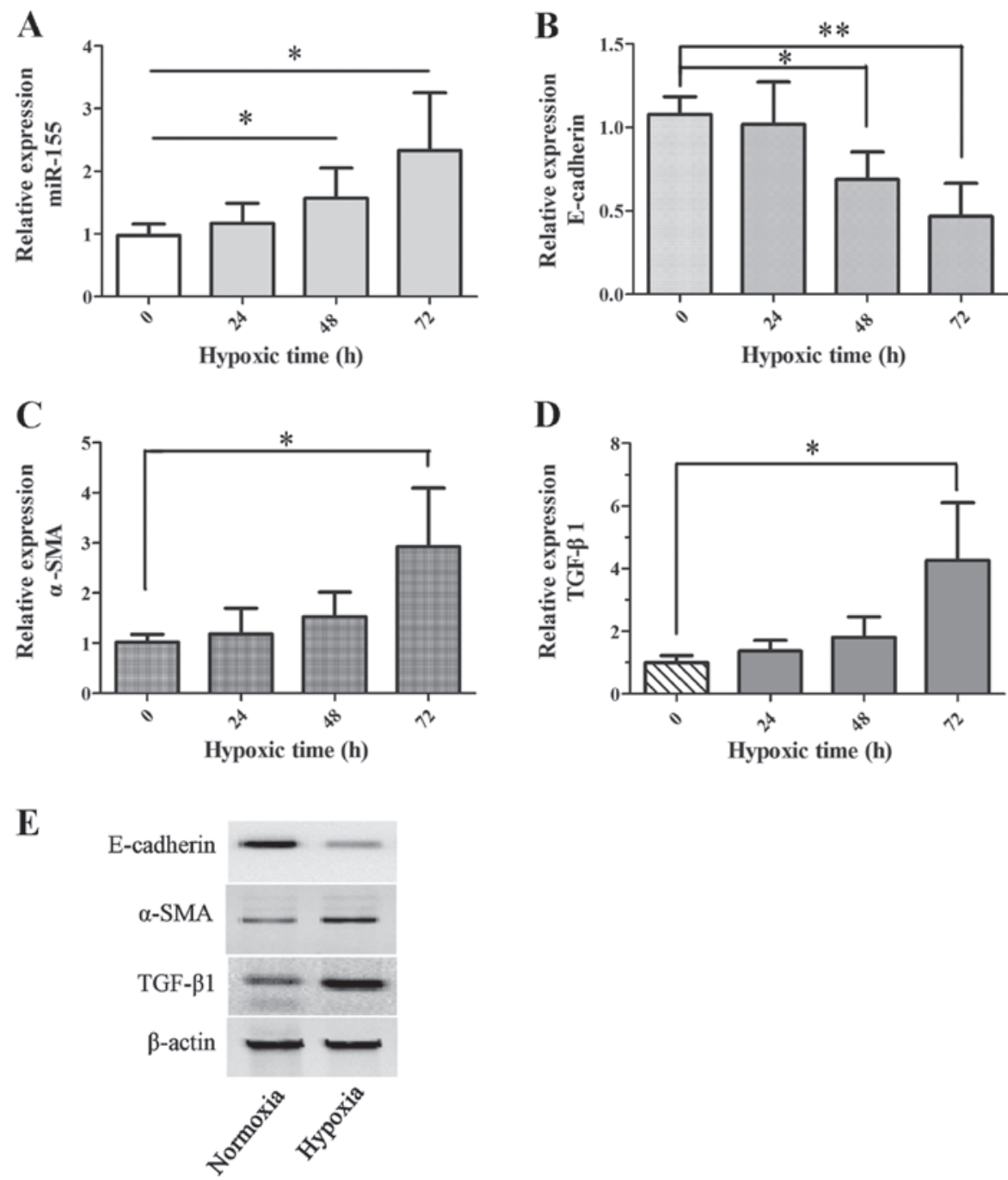

Figure 1. Comparison of the expression of miR-155 and fibrosis-associated cytokines between hypoxic and normoxic human proximal tubule (HK-2) cells. HK-2 cells were subjected to hypoxia for $0,24,48$ and $72 \mathrm{~h}$. The mRNA expression of (A) miR-155 and fibrosis-associated cytokines: (B) E-cadherin (C) alpha-smooth muscle actin ( $\alpha$-SMA) and (D) transforming growth factor beta (TGF- $\beta 1)$ was measured to analyze the differences by reverse transcription-quantitative polymerase chain reaction. U6 small nuclear RNA was used as an internal control of miR-155 and GAPDH was employed as the housekeeping control of mRNA. Data were normalized using the $2^{-\Delta \Delta C t}$ method and are expressed as the mean \pm standard deviation. " $\mathrm{P}<0.05$ and ${ }^{* *} \mathrm{P}<0.01$. (E) The expression of E-cadherin, $\alpha$-SMA and TGF- $\beta 1$ proteins in hypoxic HK-2 cells at $72 \mathrm{~h}$ was compared with that in HK-2 cells under normoxia by western blot analysis.

Significant overexpression of HIF-1 $\alpha$ mRNA was observed in hypoxic HK-2 cells at the different time points, compared with normoxic HK-2 cells (0 h; Fig. 2A). Silencing HIF-1 $\alpha$ successfully prevented augmentation in the expression of miR-155 in a hypoxic environment at $72 \mathrm{~h}(\mathrm{P}=0.031)$. However, HIF-1 $\alpha$ knockdown did not change the expression of miR-155 in normoxic HK-2 cells (Fig. 2B). Fig. 2C reveals the effectiveness of HIF-1 $\alpha$ knockdown in HK-2 cells by western blot analysis.

In addition, miR-155 mimics were transfected into HK-2 cells to investigate the effects of miR-155 on the expression of HIF-1 $\alpha$. The expression of HIF-1 $\alpha$ in HK-2 cells with transfection of the miR-155 mimics was revealed to be significantly higher than that in HK-2 cells with miR control under hypoxia at $48 \mathrm{~h}$ ( $\mathrm{P}=0.038$; Fig. 2D). All of these findings demonstrate that the expression of miR-155 was modulated in an HIF-1 $\alpha$-dependent manner in hypoxic HK-2 cells.

Overexpression of miR-155 plays a role in the process of fibrosis in hypoxic HK-2 cells. To assess the role of miR-155 in fibrosis, we investigated the expression of E-cadherin, $\alpha$-SMA and TGF- $\beta 1$ mRNA in hypoxic HK- 2 cells in which miR-155 was silenced or ectopically enhanced (anti-miR group or miR-mimics group). The expression status of miR-155 was tested, which proved the effectiveness of cell treatments as shown in Fig. 3A. Next, the expression of E-cadherin, $\alpha$-SMA and TGF- $\beta 1$ mRNA was compared among cells with miRNA control (control group), the anti-miR group and the miR-mimics group. The data revealed that the expression of $\alpha$-SMA (Fig. 3B) and TGF- $\beta 1$ (Fig. 3C) mRNA in the anti-miR group was reduced significantly at $72 \mathrm{~h}(\mathrm{P}=0.033$ and $\mathrm{P}=0.048$, respectively), whereas there was significantly augmented expression of E-cadherin mRNA at $48 \mathrm{~h}(\mathrm{P}=0.032)$ and $72 \mathrm{~h}(\mathrm{P}=0.005)$ (Fig. 3D). In the miR-mimics group, the expression of E-cadherin, $\alpha$-SMA and TGF- $\beta 1$ mRNA exhibited the opposite trend to that observed in the anti-miR group (Fig. 3B-D). All of these findings illustrate the synergistic effects of upregulated miR-155 on hypoxia-induced fibrosis in hypoxic HK-2 cells. 


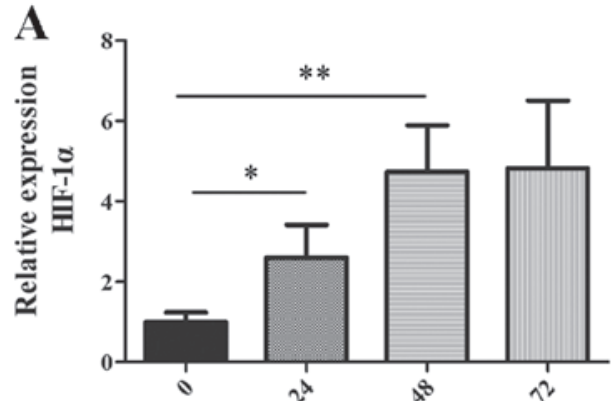

Hypoxic time (h)

C

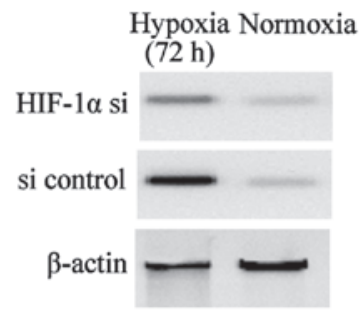

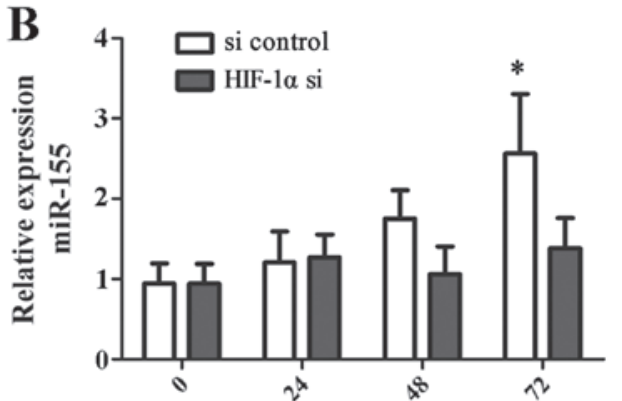

Hypoxic time (h)

D

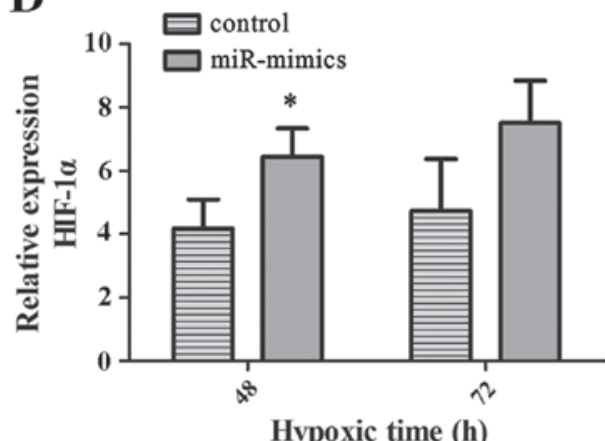

Figure 2. miR-155 is positively correlated with hypoxia-inducible factor 1 alpha (HIF-1 $\alpha$ ) in hypoxic HK-2 cells. (A) The expression of HIF-1 $\alpha$ mRNA was assayed by reverse transcription-quantitative polymerase chain reaction (RT-qPCR) in hypoxic and normoxic HK-2 cells. (B) The expression of miR-155 in cells with HIF-1 $\alpha$ knockdown (HIF-1 $\alpha$-si) was also compared with that in cells transfected with non-specific siRNA (si-control) at different time points under hypoxia. (C) Western blot analysis revealed the expression of HIF-1 $\alpha$ under hypoxia at $72 \mathrm{~h}$ and that under normoxia in the HIF-1 $\alpha$-si group and the si-control group. (D) Following treatment of cells with miR-mimics or miR control and exposure to hypoxia for 48 and $72 \mathrm{~h}$, the expression of HIF-1 $\alpha$ was assayed by RT-qPCR. "P $<0.05$ and ${ }^{* *} \mathrm{P}<0.01$.
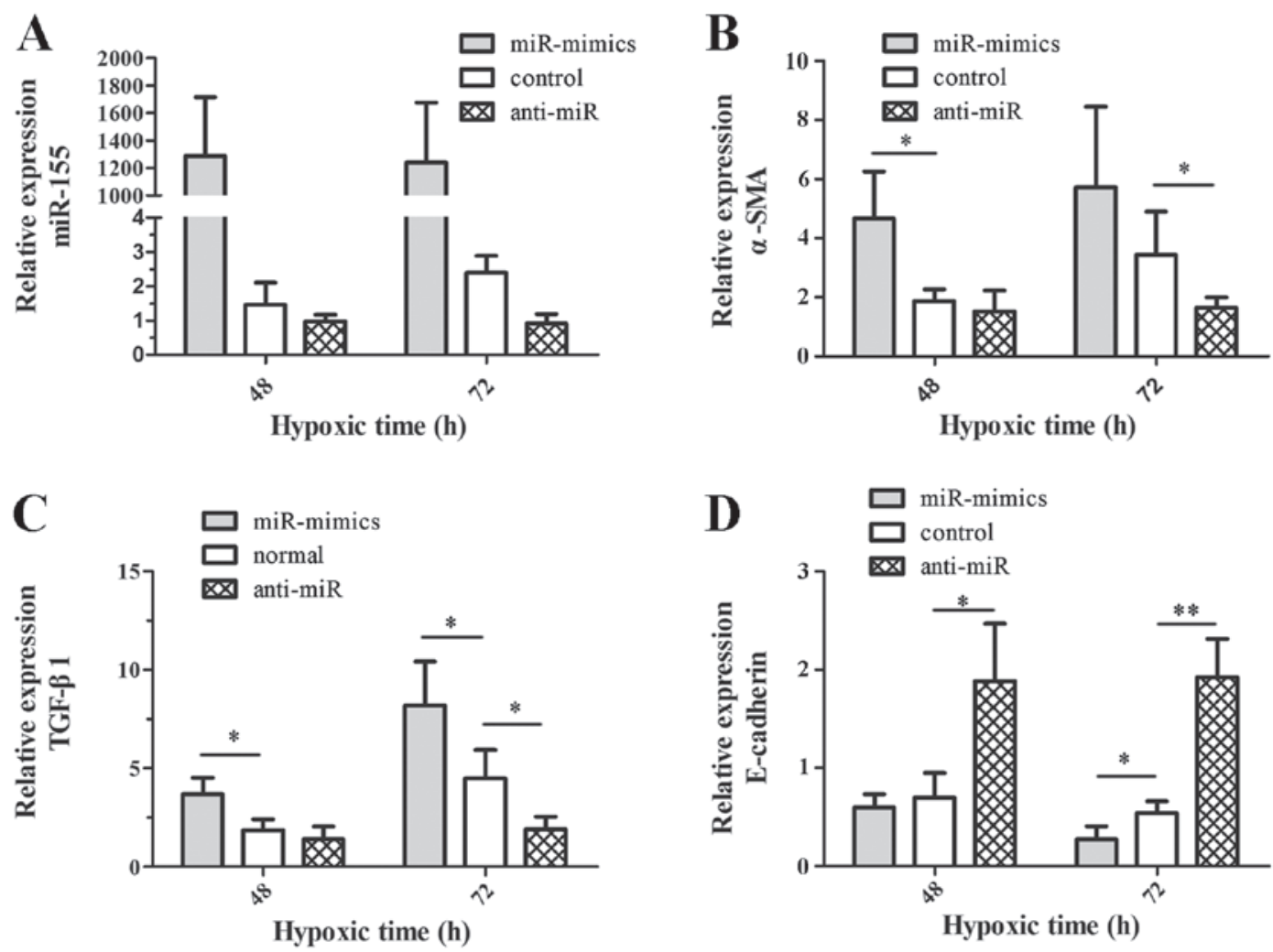

Figure 3. miR-155 regulates the expression of alpha-smooth muscle actin ( $\alpha$-SMA), transforming growth factor beta (TGF- $\beta 1$ ) and E-cadherin. (A) HK-2 cells were treated with miR-mimics $(20 \mathrm{nM})$, miRNA control $(50 \mathrm{nM})$ or anti-miR $(50 \mathrm{nM})$ and subjected to hypoxia for 48 and $72 \mathrm{~h}$. The expression of miR-155 was detected by RT-qPCR at the indicated time points. The difference in the expression of (B) $\alpha$-SMA, (C) TGF- $\beta 1$ and (D) E-cadherin mRNA was compared among the three groups. ${ }^{*} \mathrm{P}<0.05$ and $^{* *} \mathrm{P}<0.01$. 
A

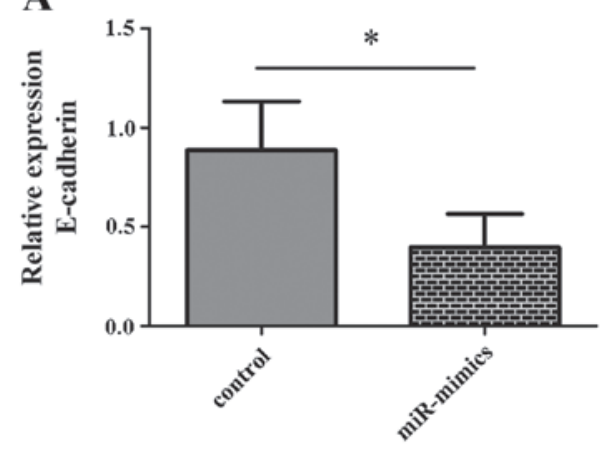

Hypoxic time 48 h

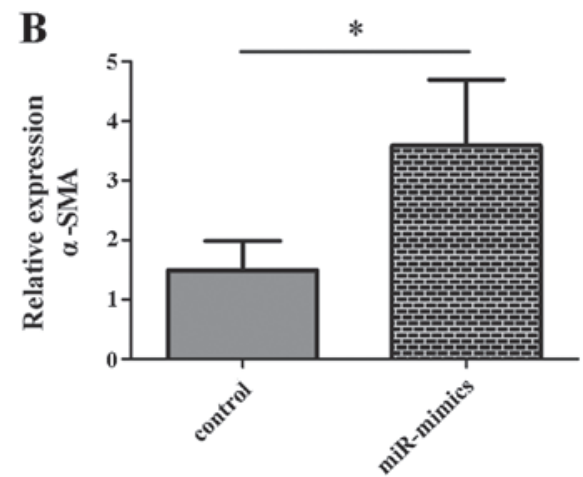

Hypoxic time $48 \mathrm{~h}$

Figure 4. miR-155 modulates the expression of E-cadherin and alpha-smooth muscle actin ( $\alpha$-SMA) independently of transforming growth factor beta (TGF- $\beta 1$ ) in hypoxic HK-2 cells. Cells were incubated with LY2157299 (TGF- $\beta$ type I receptor kinase inhibitor) under hypoxia at 48 h, following transfection with miR-mimics or miR control. The expression of (A) E-cadherin and (B) $\alpha$-SMA was compared between the miR-mimics group and the control group. ${ }^{*} \mathrm{P}<0.05$.

miR-155 modulates the expression of E-cadherin and $\alpha$-SMA independently of TGF- $\beta 1$ in hypoxic HK-2 cells. We determined whether the regulatory effects of miR-155 on the expression of E-cadherin and $\alpha$-SMA were associated with TGF- $\beta 1$, since it is a central mediator of fibrosis (14). Following treatment of cells with miR-mimics or miR control and incubation with TGF- $\beta$ type I receptor kinase inhibitor LY2157299 for $48 \mathrm{~h}$, notably decreased expression of E-cadherin mRNA ( $\mathrm{P}=0.047$; Fig. $4 \mathrm{~A})$ and increased $\alpha$-SMA $(\mathrm{P}=0.040$; Fig. 4B) was observed in the miR-mimics group (HK-2 cells in which miR-155 was ectopically enhanced), compared with the control group. These results demonstrated that upregulated miR-155 was able to promote the enhancement of $\alpha$-SMA expression and the reduction of E-cadherin expression, which were regulated by TGF- $\beta 1$. It also revealed that miR-155 modulated the process of EMT independently of TGF- $\beta 1$ in hypoxic HK-2 cells.

\section{Discussion}

Renal tissue hypoxia induces ECM production, collagen deposition and fibrosis, which are common complications of renal disease and leading causes of graft failure following kidney transplantation $(5,31)$. miR-155 has been shown to be highly correlated not only with hypoxia, but also with fibrosis of the lung and heart (29,32). Hence, we considered whether miR-155 was correlated with hypoxia-associated renal fibrosis.

In this study, we first analyzed the expression status of miR-155 and the responsiveness of proximal tubule cells to hypoxia (HK-2 cells). The results revealed significant overexpression of miR-155 and dysregulation of fibrosis-associated genes including E-cadherin, $\alpha$-SMA and TGF- $\beta 1$, which demonstrate the process of tissue fibrosis (Fig. 1). All of these findings indicated that hypoxia induced the fibrosis of HK-2 cells, and were in agreement with a review by Singh et al concerning the regulatory functions of hypoxia in fibrosis in acute kidney injury and chronic kidney disease (31). Allowing for the overexpression of miR-155, we naturally speculated that aberrant expression of miR-155 may be of significant relevance to fibrosis in hypoxic HK-2 cells.

HIF- $1 \alpha$ is a key factor in modulating the cellular response to hypoxia. Through modulation of numerous genes, HIF-1 $\alpha$ has been demonstrated to regulate pathological processes, including carcinogenesis, immunity, angiogenesis, proliferation and apoptosis (33-35). Moreover, HIF activation promotes EMT and renal fibrogenesis (36). It has also been reported that miR-155 induction contributes to a negative feedback loop for the resolution of HIF-1 $\alpha$ activity in Caco- 2 colonic epithelial cells (37). Conversely, miR-155 promotes the activity of HIF transcription factors in breast cancer (38). Therefore, the interactions of miR-155 and HIF- $1 \alpha$ may be dependent on the microenvironment, and the exact mechanism will require further investigation. In this study, we analyzed the interaction of miR-155 and HIF-1 $\alpha$ in hypoxic HK-2 cells using gain-of-function and loss-of-function approaches. It was observed that downregulation of miR-155 occurred in hypoxic HK-2 cells with HIF-1 $\alpha$ knockdown (Fig. 2B). In addition, HIF-1 $\alpha$ underwent hyper-expression due to extrinsic upregulated miR-155 in hypoxic HK-2 cells (Fig. 2D). All these results further indicated that miR-155 is positively modulated by HIF-1 $\alpha$ under hypoxia in HK-2 cells.

TGF- $\beta$ is a pro-fibrotic cytokine which is not only widely linked with the expression of ECM, but also plays central roles in EMT (2). The regulatory effects of TGF- $\beta 1$ on EMT and renal fibrosis were confirmed according to gain-of-function and loss-of-function approaches using neutralizing TGF- $\beta 1$ antibodies or ameliorating renal fibrosis in vivo and in vitro (39). $\alpha$-SMA was characterized as a biomarker for the identification of EMT and myofibroblast differentiation in tubulointerstitial fibrosis. EMT is a progressive variation in which epithelial cell adhesion (induced by E-cadherin) decreases gradually and $\alpha$-SMA increases simultaneously $(11,12,40)$. In our study, a positive effect of miR-155 on TGF- $\beta 1$ was observed (Fig. 3C). We also detected an increase of $\alpha$-SMA (Fig. 3B) mRNA and a significant reduction of E-cadherin mRNA (Fig. 3D) in the miR-mimics group. These findings revealed that miR-155 has facilitative functions on the expression of TGF- $\beta 1$ and the process of EMT. Allowing for the fundamental roles of TGF- $\beta 1$ in EMT, we aimed to clarify whether the pro-fibrotic functions of miR-155 were due to the regulation of TGF- $\beta 1$, which subsequently took effect on fibrosis, or the regulation of both TGF- $\beta 1$ and EMT. Notably, our results revealed that miR-155 modulated the expression of E-cadherin and $\alpha$-SMA independently of TGF- $\beta 1$ in hypoxic HK-2 cells (Fig. 4). In 
other words, miR-155 is capable of modulating both TGF- $\beta 1$ and the process of EMT.

In summary, these results demonstrate that hypoxia-induced miR-155 is a pro-fibrotic cytokine which is positively regulated by HIF-1 $\alpha$ in proximal tubule cells. In addition, the data also demonstrates that miR-155 promotes the fibrosis of proximal tubule cells by regulating both TGF- $\beta 1$ and the process of EMT. The results of this study may provide a new therapeutic target for prohibiting the fibrosis of hypoxic proximal tubule cells.

\section{Acknowledgements}

This study was funded by the 181st hospital of Chinese People's Liberation Army.

\section{References}

1. Higgins DF, Kimura K, Bernhardt, et al: Hypoxia promotes fibrogenesis in vivo via HIF-1 stimulation of epithelial-to-mesenchymal transition. J Clin Invest 117: 3810-3820, 2007.

2. Wynn TA: Cellular and molecular mechanisms of fibrosis. J Pathol 214: 199-210, 2008.

3. Wynn TA: Common and unique mechanisms regulate fibrosis in various fibroproliferative diseases. J Clin Invest 117: 524-529, 2007.

4. Romano E, Manetti M, Guiducci S, Ceccarelli C, Allanore Y and Matucci-Cerinic M: The genetics of systemic sclerosis: an update. Clin Exp Rheumatol 29: S75-S86, 2011.

5. Zell S, Schmitt R, Witting S, Kreipe HH, Hussein K and Becker JU: Hypoxia Induces Mesenchymal Gene Expression in Renal Tubular Epithelial Cells: An in vitro Model of Kidney Transplant Fibrosis. Nephron Extra 3: 50-58, 2013.

6. Liu Y: Cellular and molecular mechanisms of renal fibrosis. Nat Rev Nephrol 7: 684-696, 2011.

7. Li Y, Sun Y, Liu F, et al: Norcantharidin inhibits renal interstitial fibrosis by blocking the tubular epithelial-mesenchymal transition. PLoS One 8: e66356, 2013.

8. Kalluri R and Neilson EG: Epithelial-mesenchymal transition and its implications for fibrosis. J Clin Invest 112: 1776-1784, 2003.

9. Kriz W, Kaissling B and Le Hir M: Epithelial-mesenchymal transition (EMT) in kidney fibrosis: fact or fantasy? J Clin Invest 121: 468-474, 2011.

10. Barnes JL and Glass WF II: Renal interstitial fibrosis: a critical evaluation of the origin of myofibroblasts. Contrib Nephrol 169: 73-93, 2011.

11. Liu Y: New insights into epithelial-mesenchymal transition in kidney fibrosis. J Am Soc Nephrol 21: 212-222, 2010.

12. Nisticò P, Bissell MJ and Radisky DC: Epithelial-mesenchymal transition: general principles and pathological relevance with special emphasis on the role of matrix metalloproteinases. Cold Spring Harb Perspect Biol 4: a011908, 2012.

13. López-Hernández FJ and López-Novoa JM: Role of TGF- $\beta$ in chronic kidney disease: an integration of tubular, glomerular and vascular effects. Cell Tissue Res 347: 141-154, 2012.

14. Tomasek JJ, Gabbiani G, Hinz B, Chaponnier C and Brown RA: Myofibroblasts and mechano-regulation of connective tissue remodelling. Nat Rev Mol Cell Biol 3: 349-363, 2002.

15. Berk BC, Fujiwara K and Lehoux S: ECM remodeling in hypertensive heart disease. J Clin Invest 117: 568-575, 2007.

16. Lewis BP, Burge CB and Bartel DP: Conserved seed pairing, often flanked by adenosines, indicates that thousands of human genes are microRNA targets. Cell 120: 15-20, 2005.
17. Bartel DP: MicroRNAs: target recognition and regulatory functions. Cell 136: 215-233, 2009.

18. Filipowicz W, Bhattacharyya SN and Sonenberg N: Mechanisms of post-transcriptional regulation by microRNAs: are the answers in sight? Nat Rev Genet 9: 102-114, 2008.

19. Chau BN and Brenner DA: What goes up must come down: the emerging role of microRNA in fibrosis. Hepatology 53: 4-6, 2011.

20. Pinzani M, Rosselli M and Zuckermann M: Liver cirrhosis. Best Pract Res Clin Gastroenterol 25: 281-290, 2011.

21. Krenning G, Zeisberg EM and Kalluri R: The origin of fibroblasts and mechanism of cardiac fibrosis. J Cell Physiol 225: 631-637, 2010.

22. Wynn TA: Integrating mechanisms of pulmonary fibrosis. J Exp Med 208: 1339-1350, 2011

23. Wang B, Koh P, Winbanks C, et al: miR-200a prevents renal fibrogenesis through repression of TGF- $\beta 2$ expression. Diabetes 60: 280-287, 2011.

24. Zhong X, Chung AC, Chen HY, Meng XM and Lan HY: Smad3-mediated upregulation of miR-21 promotes renal fibrosis. J Am Soc Nephrol 22: 1668-1681, 2011.

25. Kato M, Zhang J, Wang M, et al: MicroRNA-192 in diabetic kidney glomeruli and its function in TGF- $\beta$-induced collagen expression via inhibition of E-box repressors. Proc Natl Acad Sci USA 104: 3432-3437, 2007.

26. Neal CS, Michael MZ, Rawlings LH, Van der Hoek MB and Gleadle JM: The VHL-dependent regulation of microRNAs in renal cancer. BMC Med 8: 64, 2010.

27. Wang H, Peng W, Shen X, Huang Y, Ouyang X and Dai Y: Circulating levels of inflammation-associated miR-155 and endothelial-enriched miR-126 in patients with end-stage renal disease. Braz J Med Biol Res 45: 1308-1314, 2012.

28. Anglicheau D, Sharma VK, Ding R, et al: MicroRNA expression profiles predictive of human renal allograft status. Proc Natl Acad Sci USA 106: 5330-5335, 2009.

29. Pottier N, Maurin T, Chevalier B, et al: Identification of keratinocyte growth factor as a target of microRNA-155 in lung fibroblasts: implication in epithelial-mesenchymal interactions. PLoS One 4: e6718, 2009.

30. Wang P, Hou J, Lin L, et al: Inducible microRNA-155 feedback promotes type I IFN signaling in antiviral innate immunity by targeting suppressor of cytokine signaling 1. J Immunol 185: 6226-6233, 2010.

31. Singh P, Ricksten SE, Bragadottir G, Redfors B and Nordquist L: Renal oxygenation and haemodynamics in acute kidney injury and chronic kidney disease. Clin Exp Pharmacol Physiol 40: 138-147, 2013.

32. Kishore R, Verma SK, Mackie AR, et al: Bone marrow progenitor cell therapy-mediated paracrine regulation of cardiac miRNA-155 modulates fibrotic response in diabetic hearts. PLoS One 8: e60161, 2013.

33. Harris AL: Hypoxia - a key regulatory factor in tumour growth. Nat Rev Cancer 2: 38-47, 2002.

34. Semenza GL: Hypoxia-inducible factors in physiology and medicine. Cell 148: 399-408, 2012.

35. Greer SN, Metcalf JL, Wang Y and Ohh M: The updated biology of hypoxia-inducible factor. EMBO J 31: 2448-2460, 2012.

36. Higgins DF, Kimura K, Iwano $M$ and Haase VH: Hypoxia-inducible factor signaling in the development of tissue fibrosis. Cell Cycle 7: 1128-1132, 2008.

37. Bruning U, Cerone L, Neufeld Z, et al: MicroRNA-155 promotes resolution of hypoxia-inducible factor 1alpha activity during prolonged hypoxia. Mol Cell Biol 31: 4087-4096, 2011.

38. Czyzyk-Krzeska MF and Zhang X: MiR-155 at the heart of oncogenic pathways. Oncogene 33: 677-678, 2014.

39. Hills CE and Squires PE: The role of TGF-beta and epithelial-to mesenchymal transition in diabetic nephropathy. Cytokine Growth Factor Rev 22: 131-139, 2011.

40. Kage $\mathrm{H}$ and Borok Z: EMT and interstitial lung disease: a mysterious relationship. Curr Opin Pulm Med 18: 517-523, 2012. 